\title{
Protective effect of Isoliquiritigenin on skin ischemia-reperfusion injury in rats
}

\author{
Guifang Zhou ${ }^{a}$, Anju Li ${ }^{b}$, Qiuling Guo ${ }^{a}$, Chuanmin Song ${ }^{c}$ and Youwen Zhang ${ }^{b}$ \\ ${ }^{a}$ Department of Oncology and Traditional Chinese Medicine, Jinan Jigang Hospital, Jinan, Shandong Province, 250132, China; \\ ${ }^{b}$ Department of Peripheral Vascular Diseases, Jinan Municipal Hospital of Traditional Chinese Medicine, Shandong Province, 250012, \\ China;'Department of Anesthesiology, Jinan Municipal Hospital of Traditional Chinese Medicine, Jinan, Shandong Province, \\ 250012 , China
}

\begin{abstract}
Skin ischemia-reperfusion (I/R) injury is a serious condition that compromises skin flap surgery and leads to organ dysfunction. It arose due to restoration of blood supply after prolonged ischemia. The present study was conducted to explore the possible protective Isoliquiritigenin (ISL) on skin ischemia-reperfusion injury in rats. The ISL was administered to rats in the dose of $10 \mathrm{mg} /$ $\mathrm{kg}, 20 \mathrm{mg} / \mathrm{kg}$, and $30 \mathrm{mg} / \mathrm{kg}$. The effect of ISL was quantified on the skin tissue of rats following the I/R injury on various indices of inflammation, oxidative stress, and apoptosis together with the effect on skin survival. ISL improves the survival of skin which is further confirmed by the histopathological analysis. It also causes a reduction of apoptosis as shown by TUNEL staining. The level of oxidative stress was found significantly reduced by restoring the MDA, SOD, and GSH levels near to normal. The level of inflammation was also decreased as suggested by reduced level of cytokines, such as TNF- $a$, IL-1 $\beta$, and IL-6. The ISL also causes a significant reduction of COX-2 and iNOS in western blot analysis. Collectively, our study has shown a beneficial effect of ISL against skin I/R injury via multiple mechanisms of action, such as attenuation of oxidative stress, inflammation, and apoptosis.
\end{abstract}

\section{ARTICLE HISTORY}

Received 27 November 2020

Accepted 21 March 2021

\section{KEYWORDS}

skin ischemia-reperfusion

(I/R) injury; isoliquiritigenin; oxidative stress;

inflammation

\section{Introduction}

The burn and trauma cause serious injuries to the skin and responsible for its deformation. It has prompted to use of plastic reconstructive surgery of skin to manage defects and physical appearance (Jeschke et al. 2008; Wang et al. 2018). Skin flap surgery has been increasingly used where healthy live tissues from one location of the body transported to the injured area that has lost skin, fat, muscle, or skeletal support. However, after translocation, flap necrosis is a serious issue affecting and compromise tissue healing (Wei et al. 2002; Hallock and Morris 2011; Ter Haar et al. 2013). Studies have suggested that ischemia-reperfusion (I/R) injury is a leading cause of surgical skin flap failure and organ dysfunction (Rah et al. 2017; Ballestín et al. 2018; Liu et al. 2019). I/R injury has been defined as a sequence of events where the loss of blood supply creates ischemia causing inconsistent exacerbation of cellular malfunction and necrosis, and subsequent restoration of blood flow to previously ischemic tissues induces reperfusion injury (Tsuji et al. 2005; Loerakker et al. 2011; Reichenberger et al. 2012). It has been assumed that reperfusion should minimize the damage, but when blood supply is restored, it increases the extent of injury due to the activity of free radicals. Studies have shown that lack of oxygen leads to the accumulation of metabolic intermediates in the tissues and with reperfusion these reactions proceed with a sudden increase in oxygen radicals (Bickers and Athar 2006; Birben et al. 2012). Accumulating shreds of evidence shows that neutrophils and inflammatory cytokines also have serious implication in the progression of ischemia/reperfusion injury (Eisenhardt et al. 2012; Donato-Trancoso et al. 2016; Romana-Souza et al. 2018). Thus, minimizing the chances of $I / R$ injury after flap surgery is still an unmet challenge and put selective pressure on the discovery of newer agents.

Isoliquiritigenin (ISL) is one of the bio-active constituents isolated from the roots of plants belonging to licorice, including Glycyrrhiza uralensis, Mongolian

CONTACT Youwen Zhang zhangyouw0806@sina.cn 
glycyrrhiza, Glycyrrhiza glabra, and so forth (Nerya et al. 2003; Chin et al. 2007). It shows wide medicinal properties, ranging from cancer to infections (Peng et al. 2015). Various studies have shown the excellent benefit of ISL against ischemic/reperfusion injury of cardiomyocytes (Zhang et al. 2013) and brain (Zhan and Yang 2006) via multiple pathways, however, no study has been conducted to determine its protective role against skin ischemic/reperfusion injury. Thus, in the present manuscript, we intend to investigate the effect of Isoliquiritigenin against skin ischemic/reperfusion injury.

\section{Methods}

\section{Establishment of animal model}

The Wistar rats after obtaining from an institutional animal house were housed in ambient temperature and humidity in pathogen-free condition with $12 \mathrm{~h}$ of alternate light and dark cycle. The rats had free access to food and water. The rats were anesthetized by intraperitoneal injection of $2 \%$ pentobarbital sodium $(20 \mathrm{ml} / \mathrm{kg})$. The rats were put into a supine position with the neck extended and the area was shaved in preparation for the procedure. The operation area was sterilized with iodophor and covered with sterile surgical towel. An axial pattern skin flap of $3 \times 6 \mathrm{~cm}$ in size was made of each rat, with the superficial artery and vein in the abdominal wall acting as the pedicle. The blood flow of the flap was blocked completely by a microvascular clip to clamp the vessel pedicle for $6 \mathrm{~h}$ and the flap was sutured to their original position with 5-0 stitches. During the period of the ischemia, anesthesia with was maintained ( $2 \%$ pentobarbital sodium). After $6 \mathrm{~h}$ of ischemia, the clamp was removed and the I/R injury model has been established.

\section{Animal groups}

The rats were divided into five groups ( $n=10 /$ group):

Group 1 Control: after the flap was harvested, it was sutured to its own donor site without clamps to reduce blood flow

Group 2 The I/R injury model was established with no treatment

Group 3 I/R injury + ISL (10 mg/kg)

Group 4 I/R injury + ISL $(20 \mathrm{mg} / \mathrm{kg})$

Group 5 I/R injury + ISL (30 mg/kg)

Rats of groups 3, 4, and 5 were treated with ISL in dosage as indicated above after suspending in 10\% Carboxy methylcellulose (CMC) by intraperitoneal injection in the right lower abdomen after the surgery, and at day 2, 4 and 6 postoperatively. The whole animal experimental design has been illustrated in Fig. 1

\section{Examination of the skin flap}

On day 7 after the operation, the survival of the dorsal flaps was assessed with the naked eye, based on color, texture, tissue elasticity, hair growth, and necrosis. The

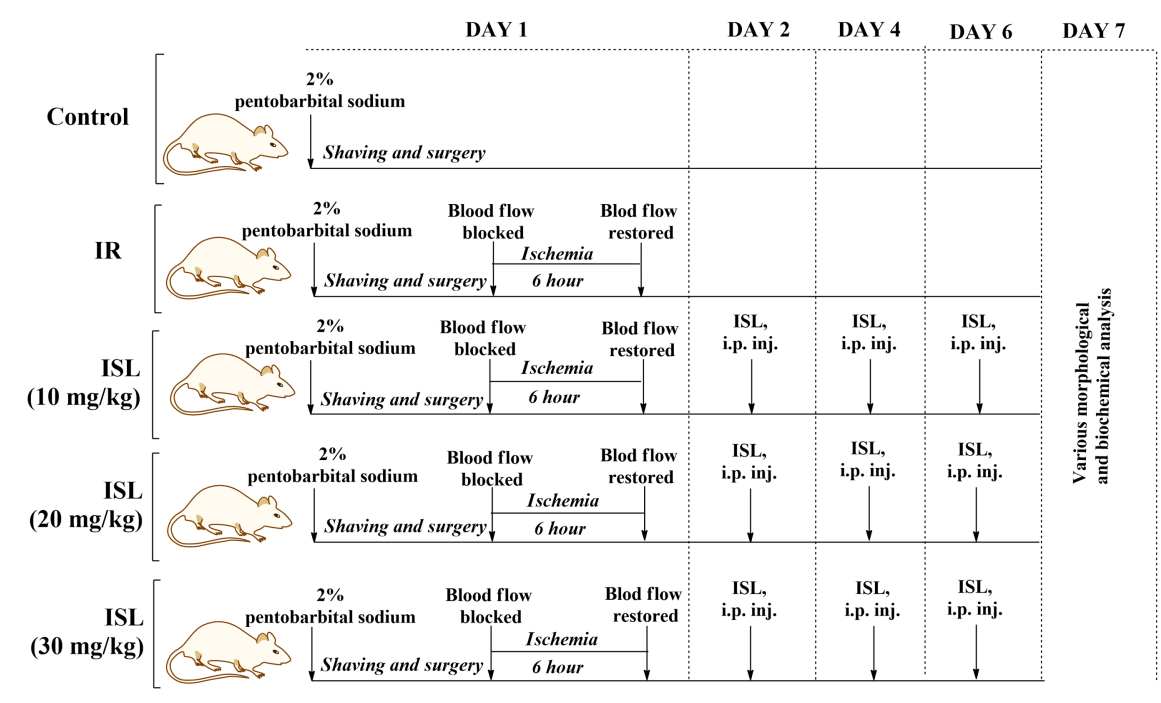

Figure 1. Experimental design of the study conducted to determine the protective of ISL against I/R injury model in rats. 
following criteria were used to determine flap necrosis: a black flap, rigid texture, shrunken tissue, poor elasticity, and no bleeding after cutting the tissue. Under anesthesia, the necrotic and surviving areas of the flaps were traced with weighing paper, pictures were taken with scale marks, and the data were imported into a computer using ImagePro Plus software V6. The survival length/width of the flap was read out, and the average survival length/width was obtained by taking 6 length/width values of each flap. The whole flap was photographed, and the effective length/width ratio of the flap after survival was calculated. The surviving area, necrotic area, and total flap area were measured with software, and the survival area ratio of the flap in the three groups was determined. Survival area ratio of the flap = (survival surface area of flap/total surface area of flap) $\times 100 \%$.

\section{Determination of flap survival}

After observation of flaps, as shown above, the images were captured via digital camera for further examination on the 7th day postoperatively. The computerized planimetry was performed using Image-Pro Plus v6.0 (Media Cybernetics, USA). The necrotic area was recorded, which was obviously different in color compared with the normal tissue. The survival rate of the flap was obtained by dividing the survival area of the skin flap by the total area of the flap.

\section{Histopathological examination}

The rats were anesthetized at 7 days postoperatively. A full-thickness skin sample of $0.5 \times 0.5 \mathrm{~cm}$ in size near the vascular pedicle was obtained and fixed in $10 \%$ formalin overnight. The sections were dehydrated in alcohol, rehydrated in xylene, and embedded in wax. $10 \mu \mathrm{m}$ consecutive section, a label for each slice, and standby, a normal slice was manufactured. The sections were stained with hematoxylin and eosin. The histological characteristics of the tissues were evaluated under a light microscope, 10 fields of view were captured per slice.

\section{TUNEL staining for apoptosis assay}

Briefly, the skin flap tissues were sectioned and dehydrated and hydrated with dehydrated alcohol and $x y-$ lene, respectively. After that, the slices were soaked in TUNEL solution at room temperature for $1 \mathrm{~h}$. Further, the slices were washed in PBS thrice for $5 \mathrm{~min}$ and fixed in paraformaldehyde containing $3 \% \mathrm{H}_{2} \mathrm{O}_{2}$ at room temperature. TUNEL staining was performed using the Roche In Situ Cell Death Detection kit for the detection of programmed cell death (Roche Applied Science, Pleasanton, CA, USA) (20). The tissue sections were then examined by microscopy (CX41RF; Olympus Corporation, Tokyo, Japan) and the number of TUNEL-positive cells was counted using National Institutes of Health $(\mathrm{NIH})$ Image software version $1.61(\mathrm{NIH}$, Bethesda, MA, USA).

\section{Evaluation of MDA, GSH, and SOD}

The level of MDA, GSH, and SOD was determined by commercially available ELISA (Jiangsu Enzyme Free Experimental Co., Ltd. China) kits as per the manufacturer protocol. The MDA levels of the tissue samples was assessed in terms of the amount of pink colour produced by the interaction of barbituric acid with MDA at high temperature and measured in an acidic media and heat $\left(90-100^{\circ} \mathrm{C}\right)$ as well as at room temperature with the aid of a spectrophotometer (Eon, Bio TeK, Winoos$\mathrm{ki}, \mathrm{VT}, \mathrm{USA}$ ) at $535 \mathrm{~nm}$. The determination of GSH uses a kinetic enzymatic recycling reaction, based on the oxidation of GSH by 5,5'-dithiobis(2-nitrobenzoic acid) (DNTB). The samples were added to the microplate wells, followed by DNTB and GSH reductase. Addition of NADPH to the wells initiated the progressive reduction of DNTB by GSH, causing a color increase that was monitored at $415 \mathrm{~nm}$. The SOD activity determined by the amount of sample that will catalyse the decomposition of $1 \mu \mathrm{mol}$ of oxygen radical $\left(\mathrm{O}^{2-}\right)$ to $\mathrm{H}_{2} \mathrm{O}_{2}$ and $\mathrm{O}_{2}$ in 1 min. The conversion of superoxide anion to $\mathrm{H}_{2} \mathrm{O}_{2}$ and $\mathrm{O}_{2}$ under enzymatic reaction conditions was applied in this kit. A spectrophotometer (Eon, Bio TeK, Winooski, VT, USA) at $420 \mathrm{~nm}$, was used to measure the chromogen colour at room temperature.

\section{Evaluation of pro-inflammatory cytokines}

The level of TNF- $\alpha$, IL-1 $\beta$, and IL- 6 were determined by commercially available ELISA kits as per the manufacturer protocol (Jiangsu Enzyme Free Experimental Co., Ltd. China). Briefly, each $10 \mu \mathrm{L}$ supernatants from control and experimental groups and $40 \mu \mathrm{l}$ sample diluents were added to the ELISA plate and incubated $30 \mathrm{~min}$ at $37^{\circ} \mathrm{C}$. The plate was washed with washing buffer five times. And then enzyme standard reagent $(50 \mu \mathrm{L})$ was added and incubated for $30 \mathrm{~min}$ followed 
by washing for five times. Finally, the chromogenic agent $\mathrm{A}(50 \mu \mathrm{L})$ and chromogenic agent $\mathrm{B}(50 \mu \mathrm{L})$ was added into each well in turns and incubated in the dark for $10 \mathrm{~min}$. Then the stop solution $(50 \mu \mathrm{L})$ was added to each well. The absorbance at $450 \mathrm{~nm}$ was measured within 15 min by using a microplate reader.

\section{Western blotting}

At 7 days after the surgery, an area of the skin flap near the vascular pedicle was harvested. The skin layer and adipose tissue were removed, and the tissue was cut into pieces and stored in a cryogenic refrigerator $\left(-80^{\circ} \mathrm{C}\right)$. RIPA lysis buffer (Beijing Solarbio Science \& Technology Co., Ltd.) containing $50 \mathrm{mmol} / \mathrm{I}$ Tris-HCl (pH 8.8), $150 \mathrm{mmol} / \mathrm{I} \mathrm{NaCl}, 1 \%$ Triton X-100, 0.1\% SDS and $1 \%$ deoxycholic acid sodium and protease inhibitors were added to the tissues (100:1), and the samples were incubated on ice, centrifuged at $12,000 \times \mathrm{g}$ for $10 \mathrm{~min}$ at $4^{\circ} \mathrm{C}$ and the supernatant extracted $30 \mathrm{~min}$ after centrifugation. The protein concentration was measured using the $B C A^{\mathrm{TM}}$ Protein assay kit (Pierce; Thermo Fisher Scientific, Inc.) according to the manufacturer's instructions. The equal amount proteins (30 $\mu \mathrm{g}$ ) were separated by $12 \%$ sodium dodecyl sulfate polyacrylamide gel electrophoresis (SDS-PAGE; Invitrogen; Thermo Fisher Scientific, Inc.) and transferred to polyvinylidene difluoride membranes (PVDF; EMD Millipore). The membranes were blocked with 5\% nonfat milk in tri-sec-buffered saline/Tween-20 (TBST) buffer (20 mM Tris-HCl, pH 7.6, $136 \mathrm{mM} \mathrm{NaCl}$ and $0.1 \%$ Tween-20). After blocking with 5\% non-fat milk for $2 \mathrm{~h}$, the membranes were incubated with primary antibodies overnight at $4^{\circ} \mathrm{C}$, and then for $2 \mathrm{~h}$ at room temperature with horseradish peroxidase-conjugated goat anti-rabbit secondary antibodies (1:5,000 dilution, ab97051, Abcam). The Pierce enhanced chemiluminescence western blotting substrate (EMD Millipore) was used to detect the signal and protein levels were quantified using Image-Pro Plus software (Media Cybernetics, Inc., MD, USA).

\section{Statistical analysis}

The data were articulated as mean \pm standard error (SEM). The statistical analysis was performed using one-way analysis of variance (ANOVA) followed by Bonferroni post hoc multiple comparison test (GraphPad Prism 5.0, USA). The P-value $<0.05$ was considered statistically significant.

\section{Results}

\section{Assessment of skin flap}

Initially, the effect of ISL was observed on the skin after induction of IR injury and results have been presented in Fig. 2. It has been found that the skin after the injury was significantly revived after the treatment of ISL in a dose-dependent manner (Fig. 2A) as compared to IR group. Moreover, in the histopathological examination, the IR model treated animals showed significant swelling and a marked increase in inflammatory cells following the IR injury as compared to control, where no such changes were observed. On the contrary, the group treated with ISL after IR showed a marked decrease in inflammatory cells. It also showed a significant reduction of edema with the restoration of tissue architecture (Fig 2B).

\section{Effect of ISL on the cellular apoptosis of skin flap}

TUNEL staining was performed to assess the antiapoptotic effect ISL, and results are presented in Fig. 3. It has been found that TUNEL positive cells were increased after IR injury as compared to control. Upon administration of ISL, the TUNEL positive cells were decreased significantly in a dose-dependent manner as compared to the IR group. These results were further elaborated by apoptosis index where ISL reduces apoptosis index as compared to control.

\section{Effect of ISL on oxidative stress}

The effect of ISL was also quantified on the indices of oxidative stress and results have been presented in Fig. 4. The control group showed no indication of oxidative stress. However, in the IR group, the level of oxidative stress has been elevated following the IR injury, as evidenced by a increased level of MDA and reduced level of SOD and GSH. However, upon administration of ISL, the level of these oxidative stress biomarkers was restored significantly near to normal in a dosedependent manner.

\section{Effect of ISL on inflammatory markers}

The effect of ISL was studied on various pro-inflammatory cytokines. As shown in Fig. 5 , the level of these cytokines (TNF- $\alpha$, IL-1 $\beta$, and IL-6) were found significantly elevated in the IR group as compared to the control. Furthermore, on the administration of ISL following the 
A

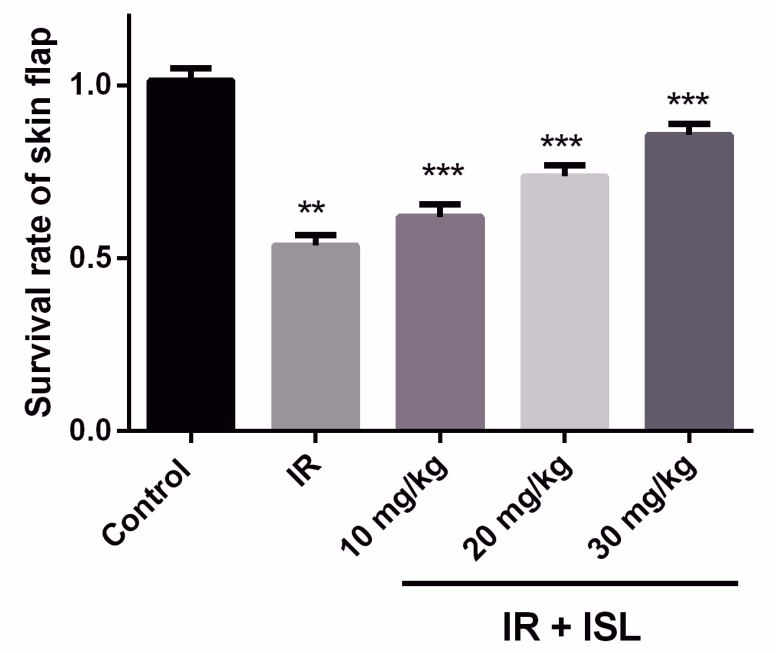

B

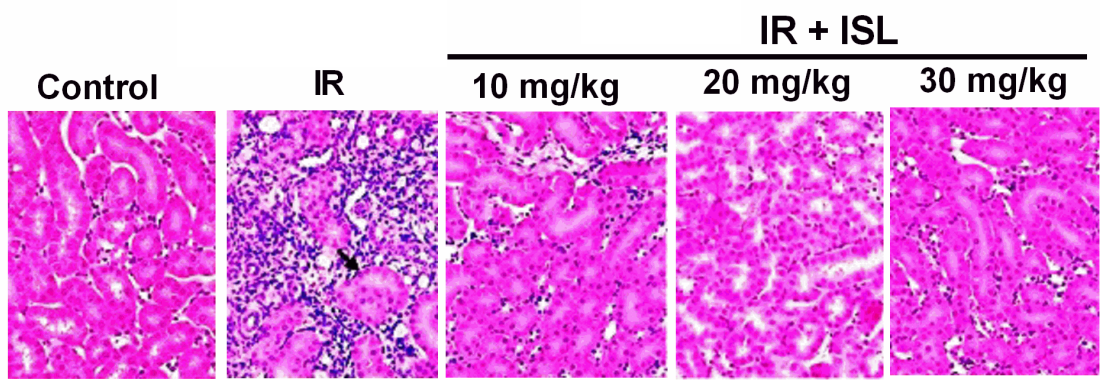

Figure 2. Effect of ISL on the (A) survival rate of skin flaps and (B) $\mathrm{H}$ and $\mathrm{E}$ staining of tissues. Values represent the mean $\pm \mathrm{SEM}$. ${ }^{* *} P<0.05$ vs control; ${ }^{* * *} P<0.05$ vs IR group, one-way analysis of variance (ANOVA) followed by Bonferroni post hoc test.

A

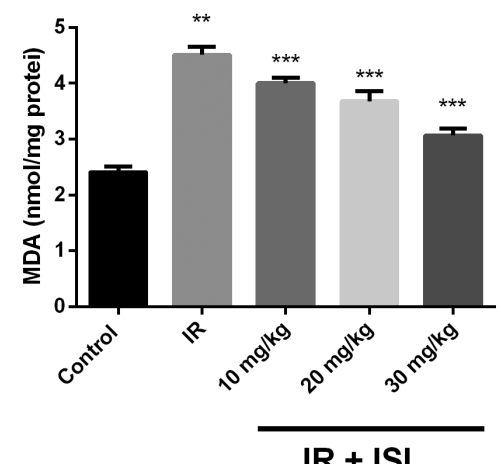

B

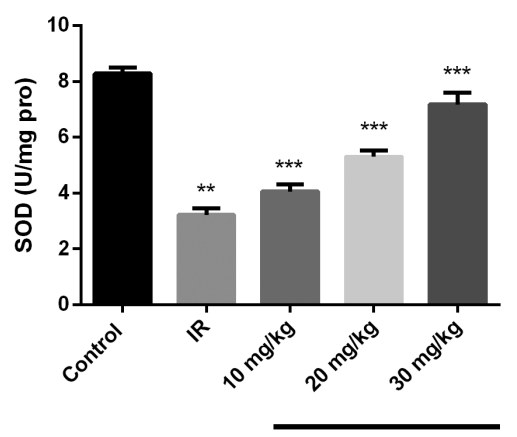

IR + ISL
C

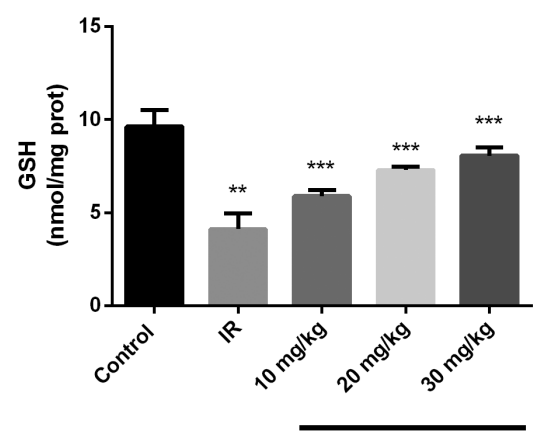

IR + ISL

Figure 4. Effect of ISL on the oxidative stress in skin IR injury in rats (A) MDA, (B) SOD, and (C) GSH. Values represent the mean \pm SEM. ${ }^{* *} P<0.05$ Vs control; ${ }^{* *} P<0.05$ Vs IR group, one-way analysis of variance (ANOVA) followed by Bonferroni post hoc test.

IR injury, the level of these tested cytokines was found significantly reduced in a dose-dependent manner.

\section{Effect of ISL on COX-2 and iNOS}

In the next instance, the effect of ISL was investigated on the expression of COX-2 and iNOS to explore its possible mechanism of action against skin IR injury via western blot analysis. As shown in Fig. 6, the level of COX-2 and iNOS was found elevated significantly in the IR group as compared to the control. Moreover, after administration of ISL, the level of these inflammatory (COX-2 and iNOS) was reduced significantly in a concentration-dependent manner. 
A

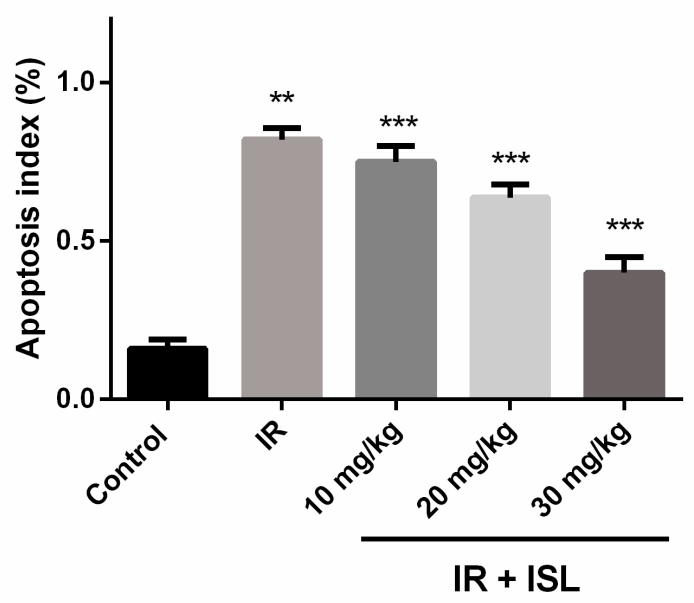

B

\section{IR + ISL}

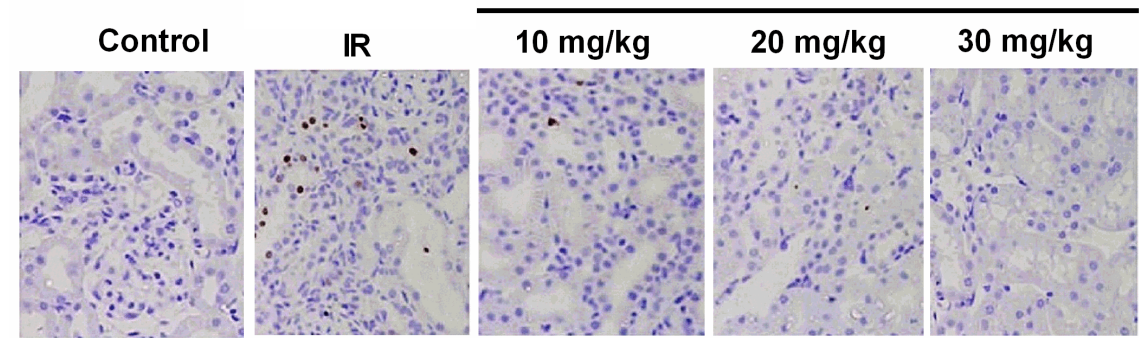

Figure 3. Effect of ISL on the apoptosis after skin I/R injury in rats (A) apoptosis index and (B) TUNEL staining. Values represent the mean \pm SEM. ${ }^{* *} P<0.05$ Vs control; ${ }^{* *} P<0.05$ Vs IR group, one-way analysis of variance (ANOVA) followed by Bonferroni post hoc test.
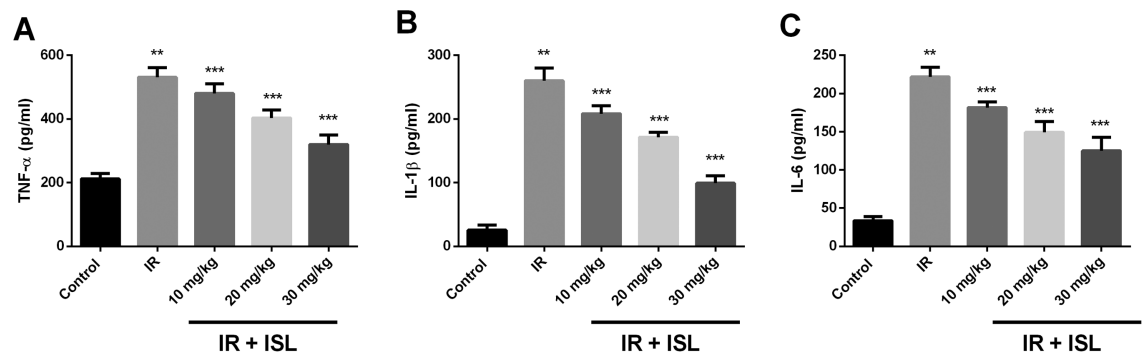

Figure 5. Effect of ISL on pro-inflammatory cytokines (A) TNF- $a_{,}(B) I L-1 \beta$, and (C) IL-6. Values represent the mean $\pm S E M$. ${ }^{* *} P<0.05$ VS control; ${ }^{* * *} P<0.05$ Vs IR group, one-way analysis of variance (ANOVA) followed by Bonferroni post hoc test.

\section{Discussion}

The skin injury poses' significant health risk if not treated well in time. It causes a significant impact on the lives of affected individuals if these injuries are arises due to burning and trauma. It markedly affects psychological wellbeing and has a pronounced effect on the daily life of the patient and associated families if the skin injury is quite deep (Ting et al. 2016). Thus, these injuries are treated efficiently by skin flap surgery where tissues from certain areas of the body are grafted on the injured site. However, during this process, the skin undergoes serious skin reperfusion injury which poses a detrimental effect on the surgery. Thus, new agents are continuously developed to tackle this situation (Hallock and Morris 2011). In our present study, we have successfully demonstrated the protective effect of ISL on the skin reperfusion injury induced in mice via skin flap surgery. This is the first report to enumerate the potential benefit of ISL against skin IR injury. The study suggested that ISL might exert its potential benefit by reducing the level of oxidative stress, inflammation, and apoptosis which results in increased survival of skin tissues. Initially, we have 


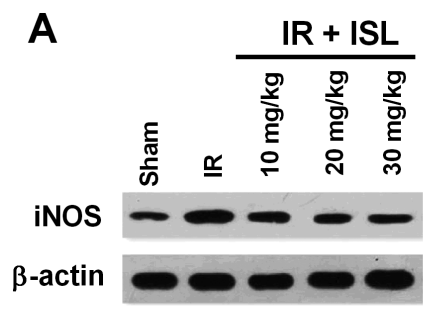

B

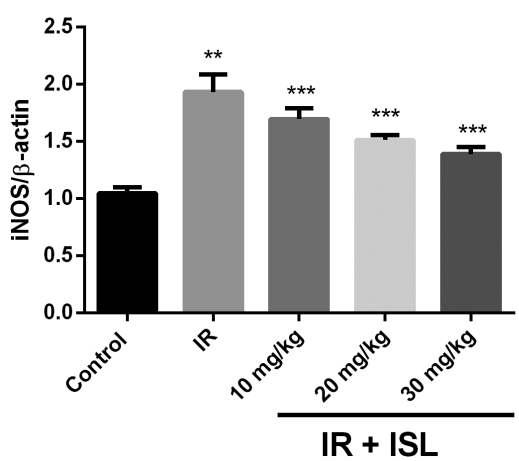

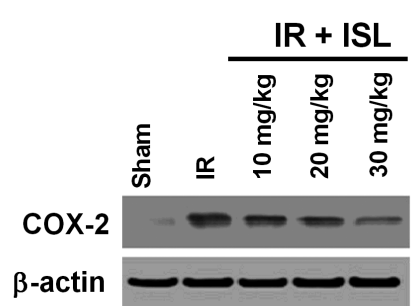

C

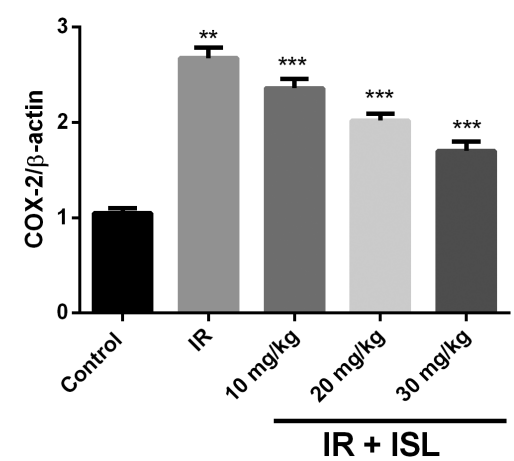

Figure 6. Effect of ISL on the expression of (A) COX-2 and iNOS by western blot, (B) qualitative bar graph showing the expression of COX2 , and (C) iNOS. Values represent the mean \pm SEM. ${ }^{* *} P<0.05$ Vs control; ${ }^{* * *} P<0.05$ vs IR group, one-way analysis of variance (ANOVA) followed by Bonferroni post hoc test.

determined the effect of ISL on the rate of survival of skin tissues after following the skin I/R injury together with histopathological analysis. It has been found that ISL causes significant revival of skin tissues possibly by further reduction of necrosis, edema, and inflammation of skin tissues as evidenced by histopathological examination. Apoptosis is the main key factor responsible for cellular death in skin grafting surgery and many ways are performed to prevent apoptosis during and after the transfer of skin tissues (Linkermann et al. 2013; Kalogeris et al. 2017). In our present study, we have shown the ISL causes a significant reduction in cellular apoptosis in TUNEL staining. Our results were found in agreement with previous studies, where ISL prevents IR injury in cardiomyocytes by reducing apoptosis (Zhang et al. 2013). Oxidative stress is another characteristic hallmark of IR injury and was induced due to the production of ROS which leads to the induction of oxidative stress led injury. It causes lipid peroxidation, DNA damage and increases vascular permeability causing tissue damage (Cuzzocrea et al. 2001; Bickers and Athar 2006; Misra et al. 2009; Ferrari and Andrade 2015). Thus, agents reducing oxidative stress have a pronounced tissue-protective effect. In the present study, ISL causes a significant reduction of oxidative stress in skin tissues by restoring the level of MDA, SOD, and GSH near to control. The ischemic reperfusion injury promotes inflammation in response to necrotic cells after ischemia and which further worsens the diseases (Huang et al. 2006; Thurman 2007; Arumugam et al. 2009; Eltzschig and Eckle 2011). Thus, it is worthwhile to assess the effect of ISL on the various pro-inflammatory cytokines. In the present study, ISL significantly reduces TNF- $\alpha$, IL- $1 \beta$, and IL- 6 following the IR injury. To further elaborate the mechanism of action of the anti-inflammatory effect of ISL, its effect was investigated on COX-2 and iNOS via western blot analysis. A study has suggested the deleterious role of both COX-2 and iNOS following the IR injury (Shinmura et al. 2002). It was showed to promote post-ischemic inflammation and actively participate in late-stage tissue destruction. Moreover, inhibition of iNOS or COX-2 showed protection against IR injury in various organs (Bolli et al. 2002; Collino et al. 2006; Candelario-Jalil and Fiebich 2008). Thus, in the present study, we examined whether ISL has an inhibitory effect on the expression of COX-2 and iNOS. It has been found that ISL has a significant inhibitory effect on the expression of COX-2 and iNOS both.

\section{Conclusion}

In summary, the results of the current study suggest that ISL exerts a protective effect against skin 
ischemia-reperfusion injury via inhibition of inflammation, oxidative stress, and apoptosis. It also causes inhibition of COX-2 and iNOS for possible strong antiinflammatory effects. Moreover, further studies are required to determine the precise mechanism of ISL against skin ischemia-reperfusion injury.

\section{Ethical Approval}

Ethical approval to report this case was obtained from the institutional animal ethical committee of Jinan Municipal Hospital of Traditional Chinese Medicine, China for biomedical experiments.

\section{Conflict of Interest}

None

\section{Funding}

The authors are thankful to The Third Batch of Jinan "Fire and Fire Inheritance 231 Project, Science and Technology Development Plan of Ji Nan Science and Technology Bureau, Subject: Review of Academic Inheritance of Traditional Chinese Medicine Surgery in Jinan (Famous Chinese Medicine Chi Jingxun School in Shandong Province) (2013-034), Shandong Province Traditional Chinese Medicine Technology Development Plan, Subject: Research on the Rule of Syndrome Differentiation and Treatment of Lower Limb Arteriosclerosis Obliterans Treated by Professor Zhang Henglong, A famous doctor of Traditional Chinese Medicine in Shandong Province (2019-0556) and Qilu Outstanding Young Talents in Health and Wellness (LWRZ-2020-3) for funding the study.

\section{References}

Arumugam T V., Okun E, Tang SC, Thundyil J, Taylor SM, WoodruffTM. Toll-like receptors in ischemia-reperfusion injury. Vol. 32, Shock. 2009. p. 4-16.

Ballestín A, Casado JG, Abellán E, Vela FJ, Alvarez V, Usón A, et al. Ischemia-reperfusion injury in a rat microvascular skin free flap model: A histological, genetic, and blood flow study. PLoS One. 2018.

Bickers DR, Athar M. Oxidative stress in the pathogenesis of skin disease. J Invest Dermatol. 2006;126:2565-75.

Birben E, Murat U, Md S, Sackesen C, Erzurum S, Kalayci O. Oxidative Stress and Antioxidant Defense. WAO J. 2012;5:9-19.
Bolli R, Shinmura K, Tang XL, Kodani E, Xuan YT, Guo Y, et al. Discovery of a new function of cyclooxygenase (COX)-2: COX-2 is a cardioprotective protein that alleviates ischemia/reperfusion injury and mediates the late phase of preconditioning. Vol. 55, Cardiovascular Research. 2002. p. 506-19.

Candelario-Jalil E, Fiebich B. Cyclooxygenase Inhibition in Ischemic Brain Injury. Curr Pharm Des. 2008;14:1401-18.

Chin YW, Jung HA, Liu Y, Su BN, Castoro JA, Keller WJ, et al. Anti-oxidant constituents of the roots and stolons of licorice (Glycyrrhiza glabra). J Agric Food Chem. 2007;55:4691-7.

Collino M, Aragno M, Mastrocola R, Benetti E, Gallicchio M, Dianzani $C$, et al. Oxidative stress and inflammatory response evoked by transient cerebral ischemia/reperfusion: Effects of the PPAR- $a$ agonist WY14643. Free Radic Biol Med. 2006;41:579-89.

Cuzzocrea S, Riley DP, Caputi AP, Salvemini D. Antioxidant therapy: A new pharmacological approach in shock, inflammation, and ischemia/reperfusion injury. Vol. 53, Pharmacological Reviews. 2001. p. 135-59.

Donato-Trancoso A, Monte-Alto-Costa A, Romana-Souza B. OIive oil-induced reduction of oxidative damage and inflammation promotes wound healing of pressure ulcers in mice. J Dermatol Sci. 2016;83:60-9.

Eisenhardt SU, Schmidt Y, Thiele JR, Iblher N, Penna V, Torio-Padron $\mathrm{N}$, et al. Negative pressure wound therapy reduces the ischaemia/reperfusion- associated inflammatory response in free muscle flaps. J Plast Reconstr Aesthetic Surg. 2012;65:640-9.

Eltzschig HK, Eckle T. Ischemia and reperfusion-from mechanism to translation. Vol. 17, Nature Medicine. 2011. p. 1391-401.

Ferrari RS, Andrade CF. Oxidative Stress and Lung Ischemia-Reperfusion Injury. Vol. 2015, Oxidative Medicine and Cellular Longevity. 2015. p. 590987.

Hallock GG, Morris SF. Skin grafts and local flaps. Plast Reconstr Surg. 2011;127:5e-22e.

Huang J, Upadhyay UM, Tamargo RJ. Inflammation in stroke and focal cerebral ischemia. Surg Neurol. 2006;66:232-45.

Jeschke MG, Chinkes DL, Finnerty CC, Kulp G, Suman OE, Norbury WB, et al. Pathophysiologic response to severe burn injury. Ann Surg. 2008;248:387-400.

Kalogeris T, Baines CP, Krenz M, Korthuis RJ. Ischemia/reperfusion. Compr Physiol. 2017;7:113-70.

Linkermann A, Hackl MJ, Kunzendorf U, Walczak H, Krautwald S, Jevnikar AM. Necroptosis in immunity and ischemia-reperfusion injury. Vol. 13, American Journal of Transplantation. 2013. p. 2797-804.

Liu H, Zhang M, Dong X, Liu Y, Hao Y, Wang Y. Necrostatin-1 protects against ischemia/reperfusion injury by inhibiting receptor-interacting protein 1 in a rat flap model. J Plast Reconstr Aesthetic Surg. 2019.

Loerakker S, Manders E, Strijkers GJ, Nicolay K, Baaijens FPT, Bader DL, et al. The effects of deformation, ischemia, and reperfusion on the development of muscle damage during prolonged loading. J Appl Physiol. 2011;111:1168-77.

Misra MK, Sarwat M, Bhakuni P, Tuteja R, Tuteja N. Oxidative stress and ischemic myocardial syndromes. Med Sci Monit. 2009;15:RA209-219. http://www.ncbi.nlm.nih.gov/ pubmed/19789524. 
Nerya O, Vaya J, Musa R, Izrael S, Ben-Arie R, Tamir S. Glabrene and isoliquiritigenin as tyrosinase inhibitors from licorice roots. J Agric Food Chem. 2003;51:1201-7.

Peng F, Du Q, Peng C, Wang N, Tang H, Xie X, et al. A Review: The Pharmacology of Isoliquiritigenin. Phyther Res. 2015;29:969-77.

Rah DK, Min HJ, Kim YW, Cheon YW. Effect of platelet-rich plasma on ischemia-reperfusion injury in a skin flap mouse model. Int J Med Sci. 2017.

Reichenberger MA, Heimer S, Schaefer A, Lass U, Gebhard MM, Germann G, et al. Extracorporeal shock wave treatment protects skin flaps against ischemia-reperfusion injury. Injury. 2012;43:374-80.

Romana-Souza B, dos Santos JS, Monte-Alto-Costa A. Caffeic acid phenethyl ester promotes wound healing of mice pressure ulcers affecting NF-kB, NOS2 and NRF2 expression. Life Sci. 2018;207:158-65.

Shinmura K, Xuan YT, Tang XL, Kodani E, Han H, Zhu Y, et al. Inducible nitric oxide synthase modulates cyclooxygenase-2 activity in the heart of conscious rabbits during the late phase of ischemic preconditioning. Circ Res. 2002;90:602-8.

Ter Haar G, Buiks SC, Kirpensteijn J. Cosmetic Reconstruction of a Nasal Plane and Rostral Nasal Skin Defect Using a Modified Nasal Rotation Flap in a Dog. Vet Surg. 2013;42:176-9.
Thurman JM. Triggers of inflammation after renal ischemia/reperfusion. Vol. 123, Clinical Immunology. 2007. p. 7-13.

Ting DSW, Cheung GCM, Wong TY. Diabetic retinopathy: global prevalence, major risk factors, screening practices and public health challenges: a review. Vol. 44, Clinical and Experimental Ophthalmology. 2016. p. 260-77.

Tsuji S, Ichioka S, Sekiya N, Nakatsuka T. Analysis of ischemiareperfusion injury in a microcirculatory model of pressure ulcers. Wound Repair Regen. 2005;13:209-15.

Wang Y, Beekman J, Hew J, Jackson S, Issler-Fisher AC, Parungao $\mathrm{R}$, et al. Burn injury: Challenges and advances in burn wound healing, infection, pain and scarring. Vol. 123, Advanced Drug Delivery Reviews. 2018. p. 3-17.

Wei FC, Jain V, Celik N, Chen HC, Chuang DCC, Lin CH. Have we found an ideal soft-tissue flap? An experience with 672 anterolateral thigh flaps. Plast Reconstr Surg. 2002;109:2219-26.

Zhan C, Yang J. Protective effects of isoliquiritigenin in transient middle cerebral artery occlusion-induced focal cerebral ischemia in rats. Pharmacol Res. 2006;53:303-9.

Zhang X, Zhu P, Zhang X, Ma Y, Li W, Chen JM, et al. Natural antioxidant-isoliquiritigenin ameliorates contractile dysfunction of hypoxic cardiomyocytes via AMPK signaling pathway. Mediators Inflamm. 2013;2013. 